\title{
Erratum: Photothermal Engineering of Graphene Plasmons [Phys. Rev. Lett. 121, 057404 (2018)]
}

Renwen Yu, Qiushi Guo, Fengnian Xia, and F. Javier García de Abajo®

(Q) (Received 27 November 2020; published 18 December 2020)

DOI: 10.1103/PhysRevLett.125.259901

A typo at the end of the second page of this Letter affects the approximate expression for the temperature-dependent graphene chemical potential. The correct expression is $\mu=E_{F}\left[\left(1+\xi^{2}\right)^{1 / 2}-\xi\right]^{1 / 2}$, with $\xi=\left(2 \log ^{2} 4\right)\left(k_{B} T_{e} / E_{F}\right)^{2}$, where $E_{F}$ is the zero-temperature Fermi energy, $T_{e}$ is the electronic temperature, and $k_{B}$ is the Boltzmann constant. Calculations in the Letter are not affected, as they are carried out using this corrected expression. 